\title{
Research of Automatic Interpretation of Analog Gauge's Indicating Data
}

\author{
Jianbo Song \\ Mailbox 1014-18 of Beijing \\ Beijing, China \\ sjbshl@163.com
}

\author{
Lei Zhang \\ Mailbox 1014-18 of Beijing \\ Beijing, China \\ sjbshl@163.com
}

\begin{abstract}
Aiming at the problem of automatic interpretation of analog instrument's dial indicating data, make the automatic interpretation solution, analyzing the relevant techniques and solving the key technical problems, so as to provide reference for realization of automatic calibration for analog meter.
\end{abstract}

Keywords- automatic interpretation; analog gauge.

\section{INTRODUCTION}

At present, instruments and gauges being calibrated automatically is a development trend of measurement detection technology. For the tested digital gauges such as digital pressure meters, digital voltmeters etc., they can be directly instructed to read data, therefore, it is easier for them to implementing automatic verification system; but, for the spring tube pressure gauges and analog voltmeters etc., their indicating data cannot be read by computer automatically, so, in test operation, the indicating data has to be read out from their dial by staff, and then in a certain way be input to semiautomated verification software. There is no doubt that this verification way affects the verification efficiency, and has the very big disparity with the automatic verification.

With the rapid development of image processing technology, the current in the image accurately uptake, pattern recognition and image information extraction and algorithm design have made great progress, which provide a possibility of automatic interpretation indicator data of the analog gauges.

After in-depth research, aiming at solving the problem of automatic interpretation analog gauges' dial data, through technical research, establishment of model, designing the dial information recognition algorithm, and using the camera equipment, including the supporting software, the analog gauges' dial data will be read out automatically, and the results are offered as a feedback to the automatic verification system, this can provide the basis for implementing automatic verification system.

In this paper, in order to solve the problem of automatically interpreting the analog meter's indicating data, the following issues are mentioned: Making analog gauge dial indicator data automatic interpretation solution; analyzing the key technical issues, and finding solutions, delivering basic analog meter data, so as to achieve to provide reference for calibrating analog gauges automatically.

\section{THE SOLUTION OF AUTOMATIC INTERPRETING THE ANALOG GAUGES’ INDICATING DATA}

A pressure gauge's dial is shown in Figure 1, the dial(1) mainly includes Center point (2), disk (4),pointer (3), dividing part reading (5), the accuracy value (7), the lower limit value (6) and upper limit value (8) etc..

According to the above discussion about automatic interpretation of analog gauge dial's indicating data, it's required to develop technical solutions to solve the relevant issues.

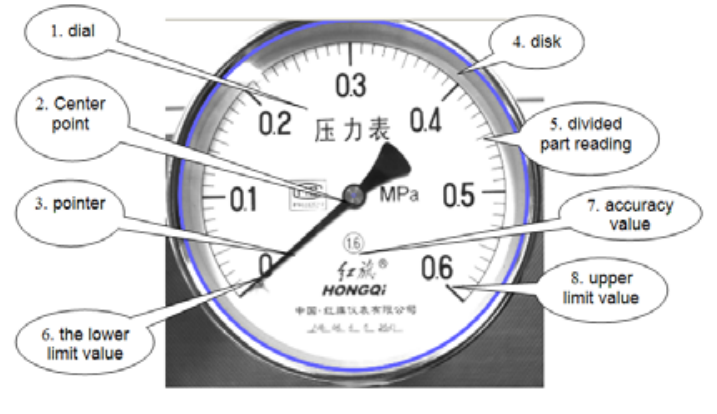

Figure 1. A pressure gauge’s dial

A. Primary issues on automatic interpretation of analog gauge dial's indicating data

According to the requirement on automatic interpretation of analog gauge dial's indicating data, some automatic reading device are developed so far, but in practice, there are still problems as following:

The first issue is how to pick up the useful information of original image. In the filming of a meter dial, the right light, accurate focus, and an enough resolution photographic equipment are all needed, at the same time the read dial should be disposed assuring clean and tidy, so as not to affect the quality of original images;

The second one is how to design the automatic interpretation algorithm which is one of the keys to achieve the task. A suitable algorithm should be offered which can ensure extracting of the center of the dial, pointer and a series of other important information, and then help to output the dial's indicating data.

The third one is how to recognizing the dial pattern accurately. Due to the differences among the types of gauge 
dials, establishing a typical dial information database is needed so as to find out the appropriate model of the corresponding dial in the pattern recognition.

\section{B. Solution of automatic interpretation of analog gauge dial indicating data}

To solve the above problems, the preliminary designing solution for automatic interpretation analog gauge dial indicating data is shown as follows.

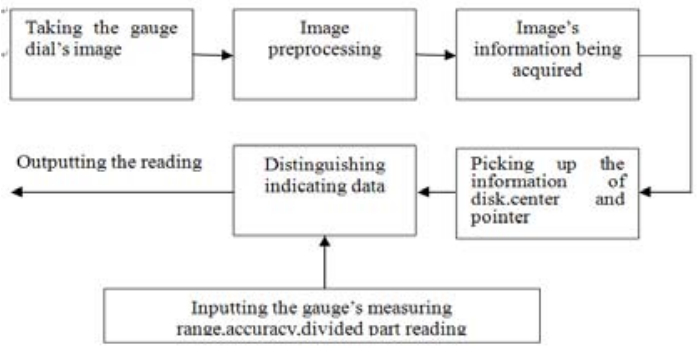

Figure 2. Solution for automatic interpretation analog gauge dial indicating data

Figure 2 shows the solution for automatic interpretation analog gauge dial indicating data, it contains the following parts:

1) Taking the gauge dial's image

Taking the gauge dial's image so as to get the digital image for computer is the primary step for automatic interpretation. by the adaptive photography equipment such as camera, the original image of dial can be obtained;

\section{2) Image preprocessing}

The image preprocessing is to preprocess the original image and judge if it is an image of an analog meter; does its brightness meet the requirements? Whether the image is complete? By image processing software the picture's position and gray degree can be adjusted, sharpening can be processed when necessary, and the outliers existing in the image being eliminated, therefore, the picture's readability will be enhanced providing the basis for accurately capturing image information.

3) Image's information being acquired

The acquisition of the image's information by image processing software provides a basis for later stage which will pick up the information of the image.

4) Picking up the information of disk, center, pointer

Extracting the information of the gauge dial's disc, center and the position of pointer is to provide a basis for calculating the indicating data.

5) Inputting the gauge's measuring range, accuracy, divided part reading

Inputting the gauge's measuring range, accuracy; divided part reading by anyway is to determine the allowable error and the tested gauge's resolution, which will offer a basis for calculating the indicating data.

6) Distinguishing indicating data

According to the obtained information about the gauge's disk, center and pointer, as well as the calculated resolution of the gauge, through the pattern recognition and interpretation algorithm, the interpretation results can be outputted. This step is a key one to interpret the indicating data of the analog meter automatically and accurately.

\section{ALGORITHM DESIGNING FOR AUTOMATIC} INTERPRETATION OF THE ANALOG GAUGE DIAL'S INDICATING DATA

Through the above analysis, "interpretation of data" is a key link in automatic and accurate interpreting of analog meter dial's indicating data. Which relates to the automatic interpretation algorithm designing, avoiding inaccurate interpretation?

\section{A. Using Hough transformation to Extract the basic information of disc and its pointer}

Hough transformation is affected by noise relatively weakly, which is adaptable for graph (such as straight, circle, elliptic shapes) edge detection and can interlink the discontinuous edge pixel due to shadows, occlusion. So, Hough transformation is used to obtain the basic information of disk and its pointer.

Detecting the edge of disk with Hough transformation can get the circle of the gauge's dial. The basic idea is to map the points in the meter image space to the parameter space for statistical calculation, so as to determine the possibility of the circle radius and center position based on the calculated results. For one point $(x, y)$ in [0,1] space the two value of instrument in the image, if it is at the circle which center is seated at the point $(m, n)$ and its radius is $r$, the corresponding parameter space meet:

$$
\left\{\begin{array}{l}
m=x-r \cdot \cos (\theta) \\
n=y-r \cdot \sin (\theta)
\end{array}\right.
$$

Among them, $\theta \in[0,2 \pi]$.In other words, in the Hough transformation, the arbitrary point $(x, y)$ in the processed image, $\Delta \theta$ and $\Delta r$ respectively traverse [ $0,2 \pi$ ] and [R1, $\mathrm{R} 2$ ], wherein R1, R2 are the circle's edge boundary. Then a parameter space $(m, n, r)$ will be obtained. $A(m, n, r)$ expresses the number of the points on the edge of the circle. The greater the value of $A(m, n, r)$, the greater of the possibility that the circle exists which radius is $r$ and center is seated at $(x, y)$. To get the correct circle, it's necessary to look for the maximum of $A(m, n, r)$

Detecting the circle with Hough transformation, the edge of the disk and the center point can be seated quickly whereas many false circles would be gotten. The treatment method is as following: in the preprocessing for smoothing, the image could be made fuzzier, and then extract the actual disk accurately with the least square method.

B. Extracting the gauge's disk and pointer accurately with the least square method

The least square method is to obtain some absolutely unknowable truth value in the simplest way, and makes the 
square sum of error to a minimum. The least square method is usually used for curve fitting.

1) Extracting the gauge's disk accurately with the least square method

In the accurate extraction for disc information, it's needed to fit circle curve with the method of least square. Figure 3 is the processing sketch map.

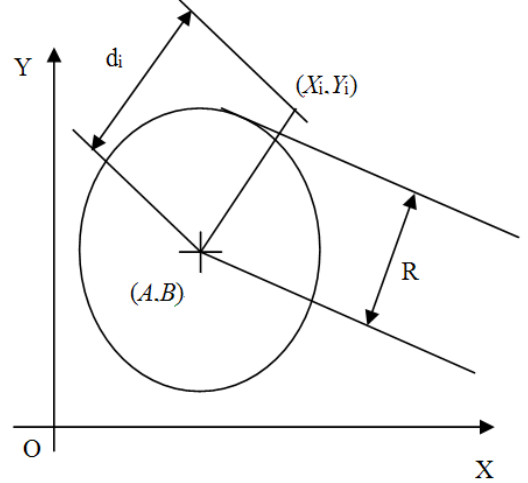

Figure 3. Sketch map of processing with the least square method for extracting disk

The basic equations of circular curve:

$$
\begin{gathered}
R^{2}=(x-A)^{2}+(y-B)^{2} \\
R^{2}=x^{2}-2 A x+A^{2}+y^{2}-2 B y+B^{2} \\
\text { Make, } a=-2 A, b=-2 B, c=A^{2}+B^{2}-R^{2}
\end{gathered}
$$

You can get:

$$
x^{2}+y^{2}+a x+b y+c=0
$$

Sample set $\left(X_{i}, Y_{i}\right)$, where $I \in(1,2,3, \ldots N)$,

Make distance from point $\left(\mathrm{X}_{\mathrm{i}}, \mathrm{Y}_{\mathrm{i}}\right)$ to the center as $d_{i}$,

$$
d_{i}^{2}=\left(X_{i}-A\right)^{2}+\left(Y_{i}-B\right)^{2}
$$

Make $\delta_{i}=d_{i}^{2}-R^{2}$, then make $\sum \delta_{i}^{2}$ as minimum, obtain a, b and c, then the needed disk information can be get.

2) Extracting the pointer information accurately with the least square method

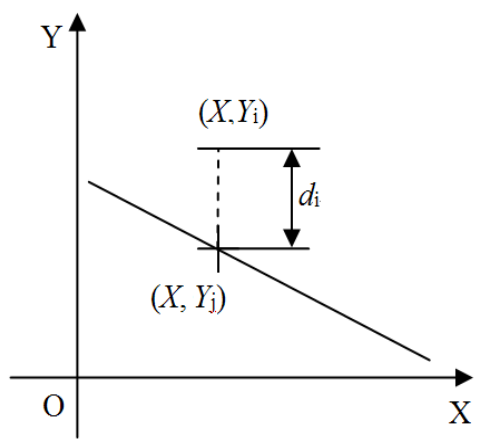

Figure 4. Sketch map of processing with the least square method for extracting pointer
In the accurate extraction for disk information, it's needed to fit line with the method of least square. Figure 4 is the processing sketch map.

The basic equations of line:

$$
y_{j}=a_{0}+a_{1} x
$$

The deviation between measured value $Y_{i}$ and Computing value

$$
Y_{i}-Y_{j}
$$

Make, $\delta_{i}=Y_{i}-Y_{j}$

Make $\sum \delta_{i}^{2}$ as minimum, obtain $\mathrm{a}_{0}, \mathrm{a}_{1}$, then the needed line can be get.

\section{Algorithm for automatic interpretation analog gauge's indicating data}

Previously, Hough transformation algorithm was mostly used to extract the pointer's position information for the static image which quality is very high. But if the image is fuzzy caused by the factors such as the pointer rocking, overlapping text etc. The detecting result will appear the obvious bias in judgment.. All in all, the automatic interpretation algorithm can be shown as figure 5 .

The first step, input the range, accuracy and divided part reading;

Secondly, extract the disk with Hough transformation method, judging whether there is any irregular in the image, if nothing is abnormal, disk information can be extracted by the least square method, and then the center of the disk is gotten;

Thirdly, the preliminary extraction for the pointer primary information is carried out by Hough transformation method, if the primary pointer information meets the request, the accuracy information of the pointer can be gotten by the least square method. Otherwise, the image should be processed where there is abnormal,

The last step, according to the determined center and pointer of the gauge's dial, combined with the range, accuracy and divided part reading, the indicating data of the analog gauge can be read out. 


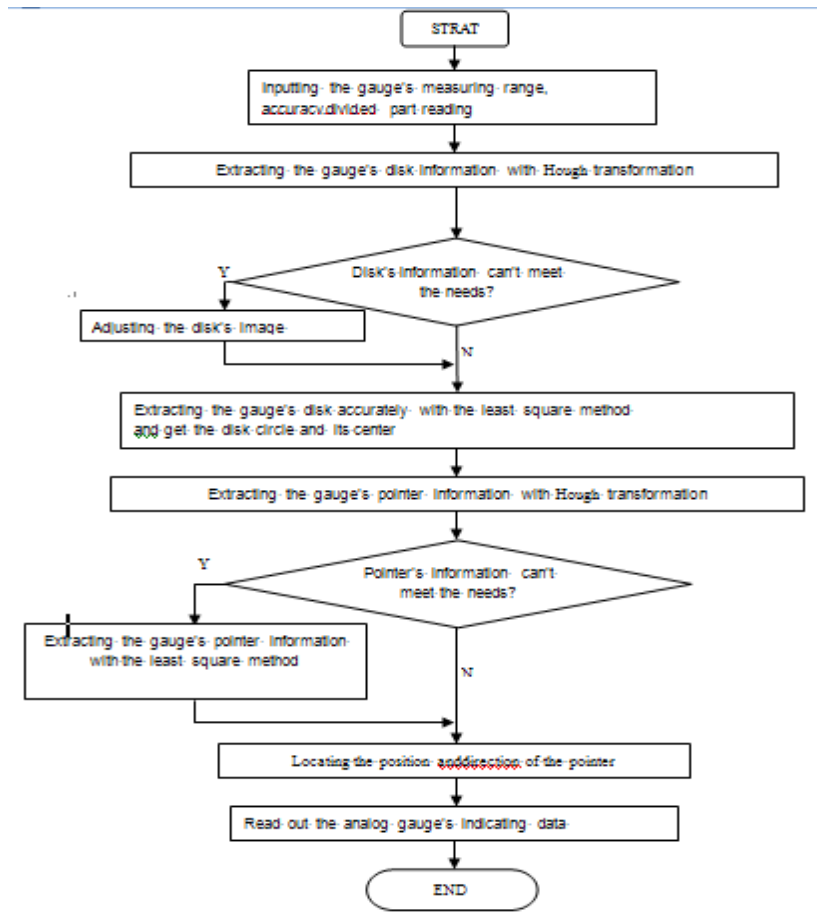

Figure 5. The automatic interpretation algorithm

\section{EFFECTION EVALUATION}

Based on the research above, the indicating data of analog gauge's dial was identified automatically while 121 images including 8 types of gauges were tested; it showed that the interpretation accuracy can reach above 99\%. The good effect has been made on the video, too.

As shown in Table 1, when an analog pressure gauge was calibrated, the gauge's indicating data was read by the manual and automatic interpretation way. The difference between the two is very small and does not exceed the allowable error basically (+ $0.05 \mathrm{MPa})$.
TABLE 1: COMPARISON THE READING RESULT BY ARTIFICIAL WAY AND THAT BY AUTOMATIC WAY

Name: general pressure gauge;

No.: 05552537;

Range: $(0 \sim 0.6) \mathrm{MPa}$

\begin{tabular}{|c|c|c|}
\hline $\begin{array}{c}\text { Standard pressure } \\
\text { value(MPa) }\end{array}$ & $\begin{array}{c}\text { Data read by } \\
\text { artificial way } \\
\text { (MPa) }\end{array}$ & $\begin{array}{c}\text { Data read by automatic } \\
\text { way (MPa) }\end{array}$ \\
\hline 0 & 0.000 & 0.000 \\
\hline 0.1 & 0.995 & 0.990 \\
\hline 0.2 & 0.295 & 0.290 \\
\hline 0.3 & 0.390 & 0.390 \\
\hline 0.4 & 0.490 & 0.495 \\
\hline 0.5 & 0.585 & 0.590 \\
\hline 0.6 & 0.690 & 0.690 \\
\hline
\end{tabular}

\section{CONCLUSION}

In this paper, combined with the actual work, the automatic interpretation algorithm and preliminary implementation effect are discussed. It's expected that the research work can be helpful for those who are engaged in the researching work in this field.

\section{REFERENCES}

[1] Xiaozhong Liao, Ru Lai,Science and Engineering. P.198,Beijing: National Defence Industry Press, January,2003

[2] H. knights, Mathematical Formula P170, Ocean Press, 1983 March

[3] SankarJayaram,HughIConnacher,KevinWLyons.Virtual Assembly Using Virtual Techniques[J].Computer Aided Design,1997,29(8)578584

[4] Bradely C,Vikers G W.Free form reconstruction for machine vision rapid prototyping.Optical Engineering,1993,32(9):2191-2200

[5] NielsonGM. Minimun Norm Interpolation Triangles[J].SIAMJournalonNumerAnal,1980,17(1):44-62

[6] LeeS,WolbergG,ShinSY.Scattered Data Interpolation with MultilevelB-splines[J].IEEE Transactional on Visualization and Computer Graghics,1997, 3(3):228-244 